\title{
Preoperative assessment of cor triatriatum in an adult by dynamic three dimensional echocardiography was more informative than transoesophageal echocardiography or magnetic resonance imaging
}

\author{
Thomas Bartel, Silvana Müller, Annette Geibel
}

\begin{abstract}
Classic cor triatriatum is rarely found in adults. Preoperative assessment of classic cor triatriatum in a 22 year old man without symptoms by three dimensional echocardiography was more informative than transoesophageal echocardiography or magnetic resonance imaging, which both showed only a small hole in the membrane separating the accessory atrium from the true left atrium. The size of the hole indicated a strong likelihood that symptoms would develop in this patient. Dynamic three dimensional echocardiography, however, showed a long wide hole in the separating membrane. This finding was consistent with the absence of symptoms in this patient. Symptom free patients with moderate obstruction do not need early surgical correction.
\end{abstract}

(Br Heart f 1994;72:498-499)

In cor triatriatum the pulmonary veins enter an enlarged common pulmonary vein that is shaped like a windsock and is sometimes described as an accessory left atrium. This accessory chamber communicates with the hypoplastic but true left atrium through a narrow opening. The accessory atrium may communicate directly or indirectly with the

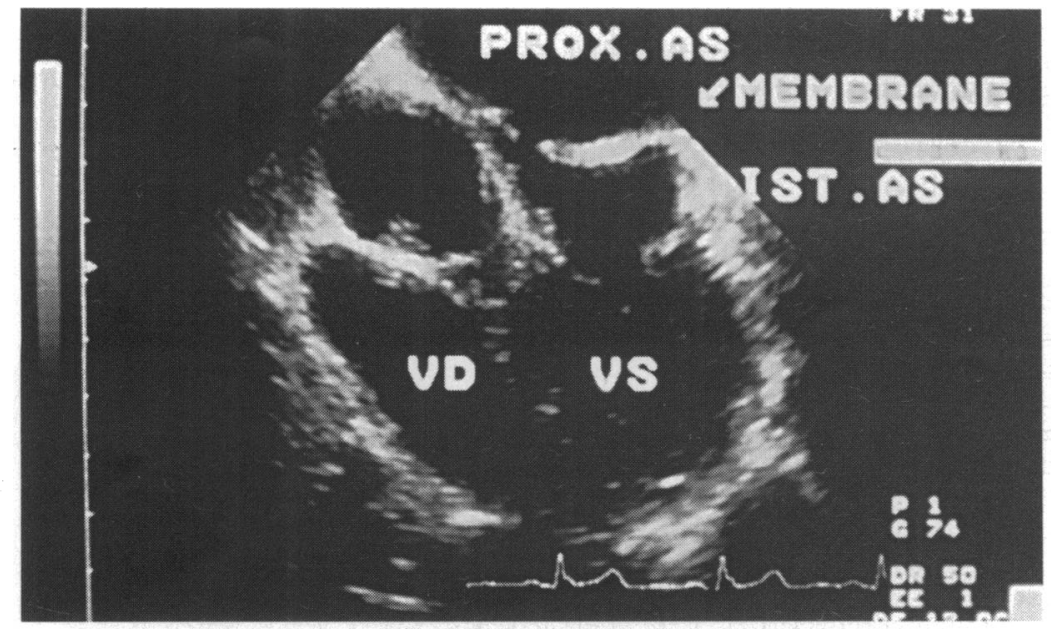

Figure 1 Transoesophageal echocardiography showing a small hole in the separating membrane. $V D$, right ventricle; $V S$, left ventricle; prox $A S$, accessory atrium; dist $A S$, true left atrium. right atrium by way of an anomalous channel. In classic cor triatriatum, however, a membranous partition, which is regarded as the wall of the dilated common pulmonary vein, separates the accessory chamber from the true left atrium. The true left atrium communicates with the left ventricle through the mitral valve. In most patients severe symptoms develop within the first few years of life. If there is a large enough hole in the membranous partition, however, patients can remain symptom free until they are adult.

\section{Case report}

A well developed 22 year old man presenting with normal exercise tolerance was admitted for evaluation of mild recurrent bronchitis and a noisy, pandiastolic murmur, which was heard best over the third intercostal space in the left parasternal line. The electrocardiogram, which showed incomplete right bundle branch block but no atrial fibrillation, indicated isolated, moderate right heart overload. The chest radiograph showed a slightly enlarged left atrium. Transthoracic echocardiography established the diagnosis of cor triatriatum, which was confirmed by monoplane transoesophageal echo (TOE) and magnetic resonance imaging (MRI). In addition cardiac catheterisation showed that the systolic right ventricular pressure was $39 \mathrm{~mm} \mathrm{Hg}$. TOE and MRI showed a narrow hole (about $9 \mathrm{~mm}$ in diameter) in the membranous partition adjacent to the left ventricular outflow tract (fig 1). No atrial septal defect was detected.

A staged operation was considered, because it was expected that progressive congestion followed by cardiac decompensation would develop in the next few years. Because the patient's lack of symptoms did not accord with the malformation shown by TOE and MRI we examined him by dynamic three dimensional echocardiography. We used the TomTec-Echo-Scan and Echo-CT-TEE probe for parallel scanning of the heart. Digital data for subsequent three dimensional image reconstruction were obtained. This method showed the full extent of the opening in the membrane, which surprisingly ran the whole length of membranous partition and was not a narrow hole as suspected before (fig $2 \mathrm{a}$ and $\mathrm{b}$ ). The opening was about $25 \mathrm{~mm}$ long and $10 \mathrm{~mm}$ wide. Dynamic three 
Figure 2 The true extent of the hole in the membrane separating the accessory atrium from the true left atrium was shown only by three dimensional echocardiography. (1) Separating membrane, (2) true left atrium, (3) accessory left atrium, (4) hole.
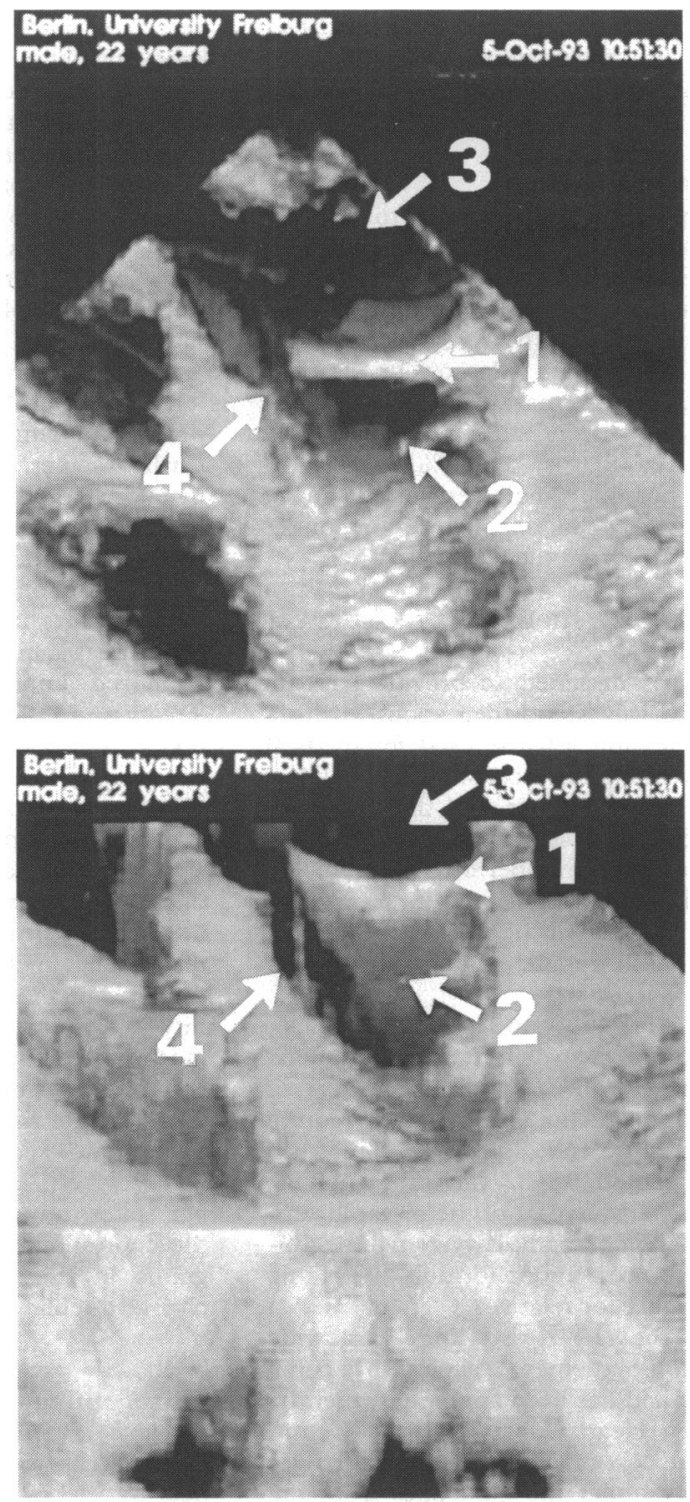

dimensional echocardiography showed that the separating membrane moved like an additional valve with low grade stenosis.

\section{Discussion}

In adults with classic cor triatriatum who are symptom free or only mildly affected the prognosis and the urgency of surgical correction have been difficult to establish because cardiac decompensation can progress rapidly after a long symptom free period. ${ }^{1}$ Until recently either TOE or MRI has been regarded as the best way of imaging cor triatriatum in adults. ${ }^{2}$ Most reports regard TOE as the most practicable diagnostic approach. ${ }^{3-5}$ Electrocardiographic evidence of right ventricular overload is a typical finding in cor triatriatum and the electrocardiogram is a valuable way of distinguishing between cor triatriatum and mitral stenosis. Atrial fibrillation and a broad, notched $P$ wave do not occur in cor triatriatum before cardiac decompensation, because the accessory atrium is electrocardiographically inactive and the true left atrium is small. ${ }^{2} \mathrm{We}$ believe that ours is the first report of the use of dynamic three dimensional echocardiography in classic cor triatriatum. The anatomy of the heart and especially the region of interest were accurately visualised by three dimensional image reconstruction from digital data, which were recorded with a new transoesophageal probe from about 100 tomograms. ${ }^{6}$ Though TOE and MRI showed that the windsockshaped common pulmonary vein displaced the true left atrium towards the left ventricle, thus explaining the diastolic murmur, they did not accurately show the size and the shape of the communication between the accessory and the true left atrium. TOE and MRI showed a single small hole in the membrane between the accessory atrium and the true hypoplastic left atrium. This suggests the likelihood of symptoms developing. Whereas the long, wide hole in the separating membrane, discovered by dynamic three dimensional echocardiography, could well explain the patient's history. A single and sufficiently long and wide communication allowing for normal development and preventing severe complications is rarely found. An asymmetrical windsock-shaped accessory atrium communicating with the true left atrium by a long and wide crevice has not been described before. A patient with several small defects leading to a long symptom free history, however, has been reported. ${ }^{7}$

Cor triatriatum that only becomes manifest in adulthood has been reported in nine other cases. As in severe obstruction, the patient's quality of life and load capacity can remain normal until the sixth decade and life expectancy is not necessarily reduced. ${ }^{8}$ Early surgical correction is not indicated in symptom free patients with moderate obstruction. Dynamic three dimensional echocardiography is the only diagnostic approach that shows the spatial relation between the accessory atrium and the orifice in the separating membrane. It provides additional information to cardiologists and surgeons by showing how the malformation is related to the residual parts of the heart. As in other studies, ${ }^{27}$ cardiac catheterisation did not add substantially to the information obtained non-invasively. Dynamic three dimensional echocardiography should be used to assess cor triatriatum before operation because this rare malformation is very variable.

1 Feld H, Shani J, Rudansky HW, Rudyuk E, Greengard A. Initial presentation of cor triatriatum in a 55-year-old woman. Am Heart $\mathcal{F}$ 1992;124:788-91.

2 Bartel T, Sandring KH, Schönfeld G, Müller S, Baumann G. Classic cor triatriatum: optimal imaging by transesophageal echo and magnetic resonance tomography in the adult. Am $\mathcal{F}$ Noninv Cardiol 1993;7:308-10.

3 Vuocolo LM, Stoddard MF, Lonaker RA. Transesophageal two-dimensional and Doppler echocardiographic diagnoses of cor triatriatum in the adult. $A m$ Heart $\mathcal{F} 1992 ; 124: 791-3$

4 Hoffmann R, Lambertz $H$, Flachskampf FA, Hanrath $P$. Transoesophageal echocardiography in the diagnosis of cor triatriatum; incremental value of color Doppler. Eur Heart $\mathcal{f} 1992 ; 13: 418-20$.

5 Schluter M, Langstein BA, Thier W. Transesophageal two-dimensional echocardiography in the diagnosis of cor triatriatum in the adult. $\mathcal{F}$ Am Coll Cardiol 1983; 2:1011-5.

6 Pandien NG, Nanda NC, Schwartz SL, Fan P, Cao Q, Sanyal $R$. Three-dimensional and four-dimensional transesophageal echocardiographic imaging of the heart and aorta in humans using a computed tomographic and aorta in humans using a computed tomogra

7 De Belder MA, Argano B, Burrell CJ. Cor triatriatum sinister, not mitral stenosis, in an adult with previous ister, not mitral stenosis, in an adult with previous ment by cross sectional echocardiography. Br Heart $\mathcal{f}$ ment by cross

8 Patel AK, Ninneman RW, Rahko PS. Surgical resection of cor triatriatum in a 74-year-old man. $\mathfrak{f} \mathrm{Am} S o c$ Echocardiogr 1990;3:402-7. 